\title{
Processed Product Identifier
}

National Cancer Institute

\section{Source}

National Cancer Institute. Processed Product Identifier. NCI Thesaurus. Code C95381.

A unique symbol that establishes the identity of the processed product. 